\title{
The Wide Field Monitor onboard the Large Observatory For X-ray Timing (LOFT)
}

\section{Y. Evangelista*}

IAPS-INAF, Via del Fosso del Cavaliere 100 - 00133 Rome, Italy

INFN, Sez. Roma Tor Vergata, Via della Ricerca Scientifica 1 - 00133 Rome, Italy

Email: yuri.evangelista@iaps.inaf.it

\section{on behalf of the LOFT consortium ${ }^{\dagger}$}

The Large Observatory for X-ray Timing (LOFT) is one of the four candidate ESA M3 missions considered for launch in the 2022-2024 time frame. It is specifically designed to perform fast Xray timing and to probe general relativity at extreme field strengths near black holes and neutron stars and probe matter at supranuclear densities in neutron stars. The LOFT scientific payload is composed of the Large Area Detector (LAD) and the Wide Field Monitor (WFM). Here we describe the LOFT WFM, a compact and light-weight coded mask instrument with a detection plane based on the large-area Silicon drift detector (SDD) technology. The instrument is designed to cover a large FoV in the same energy range as the Large Area Detector, namely almost 50\% of the sky in $2-80 \mathrm{keV}$ (2-50 keV of nominal energy range and 50-80 keV of extended energy range). Its design would allow the detection of transient events with fluxes down to a few mCrab per day, with unprecedented spectral resolution for a Wide Field Monitor (better than $300 \mathrm{eV}$ at $6 \mathrm{keV}$ ), a timing resolution of about $10 \mu \mathrm{s}$ and an angular resolution of 4.7 arcmin, which translates into a sub-arcmin point-source location accuracy (PSLA) for high significance $(>10 \sigma)$ detections. Such performance will allow us to trigger LAD observations of the most interesting sources, and to study long-term variability of LMXBs, HMXBs and some AGNs, and to detect and localize GRBs. Moreover, the WFM is equipped with the LOFT Burst Alert System (LBAS), an onboard trigger and imaging system with a fast-link $(<30 \mathrm{~s})$ transmission of the position of fast transients.

An INTEGRAL view of the high-energy sky (the first 10 years) - 9th INTEGRAL Workshop and celebration of the 10th anniversary of the launch

15-19 October 2012

Bibliotheque Nationale de France, Paris, France

\footnotetext{
* Speaker.
} 


\section{Introduction}

The LOFT Wide Field Monitor (WFM, see [1, 2, 3]) has the capability to simultaneously observe $1 / 3$ of the sky ( $\sim 44 \%$ at zero response, and $\sim 33 \%$ at $20 \%$ of peak response) in the same energy band as the LOFT Large Area Detector (LAD). The main purpose of the WFM is to detect sources for follow-up observations with the LAD, so the field of view is designed to have a maximum overlap with the sky accessible to LAD pointings. These sources are new transients, as well as known sources undergoing spectral state changes. The WFM is classical coded mask imaging instrument with a point source location accuracy of $1 \mathrm{arcmin}$, matching the required on-target pointing accuracy for LAD observations. The coded mask design is successfully employed by several instruments on past and on-going X-ray missions, like Beppo-SAX, INTEGRAL, AGILE, and SWIFT. The WFM has a modular design with 5 Camera Units with partly overlapping fields of view. The WFM detector employs the same basic detector technology as the LAD, but with a read-out scheme that enables $2 \mathrm{D}$ position determination of the X-ray interactions required for the imaging. However, the detector position resolution in one direction is much finer $(<100 \mu \mathrm{m})$ than in the other direction $(<8 \mathrm{~mm})$. This is reflected in the elongated point spread function of the de-convolved sky images. Therefore each of the 5 WFM Camera Units consists of two individual Cameras with a relative rotation of $90^{\circ}$, that enables to achieve the required $1^{\prime}, 2 \mathrm{D}$ position accuracy.

\section{Instrument design}

In a coded aperture imaging instrument the mask shadow recorded by the position-sensitive detector can be deconvolved by using the proper procedures to recover the image of the sky, with an angular resolution given, as a first approximation, by the ratio between the mask element and the mask-detector distance. In order to avoid losing imaging sensitivity, the mask element should not be smaller than twice the detector resolution element [4]. Proper performance of a coded-mask camera requires that every sky position is encoded on the detector in a unique way, in order to have an auto-correlation function of the mask pattern which consists of a single peak and flat side-lobes.

The WFM Camera coded mask is made of a $150 \mu \mathrm{m}$ thick Tungsten foil and its pattern consists of $1040 \times 16$ elements of dimensions $250 \mu \mathrm{m} \times 14 \mathrm{~mm}$ with $2.4 \mathrm{~mm}$ spacing between the elements in the coarse resolution direction. The mask open fraction is chosen to be $\sim 25 \%$. The detectormask distance is $202.9 \mathrm{~mm}$ to achieve the required angular resolution. The size of the mask is $\sim 1.7$ times larger than the detector, in order to achieve a flat (i.e., fully coded) region in the centre of the FoV. In the LOFT WFM we define:

- Camera: the assembly of a detector plane with its own collimator and mask, providing a fine (few arcmins) angular resolution in one coordinate and a coarse (few degrees) in the orthogonal direction

${ }^{\dagger}$ L. Alvarez, L. Amati, P. Azzarello, D. Barret, G. Bertuccio, E. Bozzo, S. Brandt, C. Budtz-Jørgensen, R. Campana, A. Cros, E. del Monte, I. Donnarumma, M. Feroci, J.L. Galvez Sanchez, D. Götz,F. Hansen, J.W. den Herder, M. Hernanz, R. Hudec, J. Huovelin, D. Karelin, S. Korpela, C. Labanti, N. Lund, C. Motch P. Malcovati, P. Orleanski, E. Perinati, M. Pohl, A. Rachevski, A. Santangelo, S. Schanne, C. Schmid, S. Suchy, C. Tenzer, A. Vacchi, J. Wilms, G. Zampa, N. Zampa, J.J.M. in 't Zand, A. Zdziarski. 
- Camera Unit: a set of 2 Cameras oriented at $90^{\circ}$, covering the same field of view. The use of the 2 Cameras as a Unit enables a fine 2D angular resolution

- WFM Assembly: the total set of Cameras composing the WFM, covering the entire field of view with fine angular resolution.

In Table 1 we summarize the Camera design.

\begin{tabular}{l|c} 
Parameter & Value \\
\hline Concept & Coded mask imager \\
Mask thickness & $150 \mu \mathrm{m}$ \\
Mask material & Tungsten \\
Mask open fraction & 0.25 \\
Mask-detector distance & $202.9 \mathrm{~mm}$ \\
Mask size & $260 \times 260 \mathrm{~mm}^{2}$ \\
Mask pitch & $250 \mu \mathrm{m} \times 16 \mathrm{~mm}$ \\
On-axis effective area (@6 keV) & $41 \mathrm{~cm}^{2}$ \\
\hline
\end{tabular}

Table 1: WFM Camera design.

The whole WFM is composed of 5 Camera Units (10 Cameras, see Figure 1), 5 Back-End Electronics and 1 Data Handling Unit. The Back-End Electronics and Power Supply Unit (providing low, medium and high voltages power lines) for each Camera Unit are collected into a single electronics box, located close $(<1.5 \mathrm{~m})$ to the Camera Units. Each Camera is composed of 1 Detector Tray, 4 Silicon Drift Detectors, 4 Front-End Electronics, 4 Be layers, 1 Coded Mask with a Thermal Blanket, 1 Collimator. The Units and the Cameras in the WFM are organised to achieve a high level of redundancy.
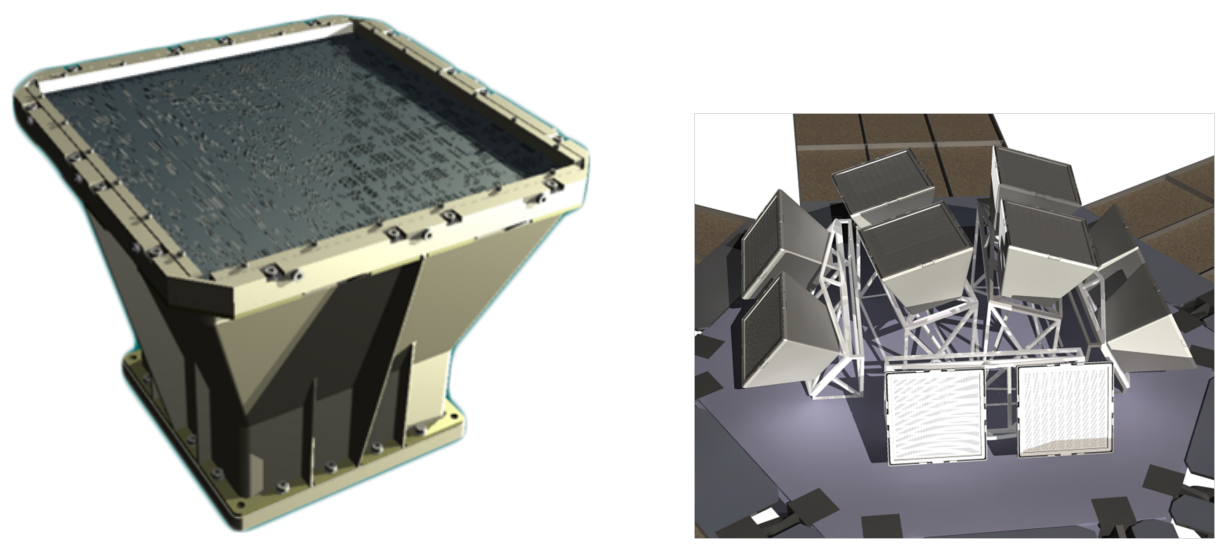

Figure 1: Mechanical design (left) of a WFM Camera. The overall LOFT WFM (right) is composed by 5 Units, each one made of 2 Cameras with the fine resolution directions orthogonally arranged.

\subsection{Detectors}

The WFM design is based on the SDD detectors. The detectors of the WFM are designed to optimise their imaging properties, when combined with the coded mask. The general working 

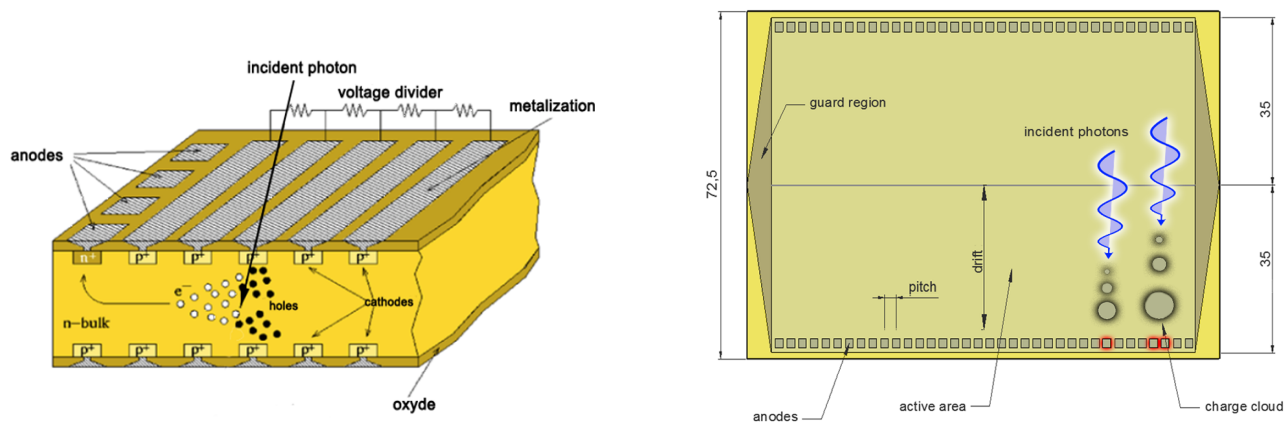

Figure 2: Working principle of the large-area Silicon Drift Detectors.

principle of the large-area SDD detector is described in Figure 2. The electron cloud generated by the X-ray photon in the Silicon bulk (Figure 2, left) is drifted towards the anodes by means of a constant electric field. The width of the Gaussian cloud, and thus the number of collecting anodes, is a function of the photon absorption point $(x)$ in the drift direction, according to

$$
\sigma=\sqrt{2 D t+\sigma_{0}^{2}}=\sqrt{2 \frac{k_{B} T}{q} \mu \frac{x}{\mu E}+\sigma_{0}^{2}}=\sqrt{2 \frac{k_{B} T}{q E} x+\sigma_{0}^{2}}
$$

where $D$ is the diffusion coefficient, $t$ is the drift time, $k_{B}$ is the Boltzmann's constant, $\mu$ is the electron mobility, $T$ is the absolute temperature and $\sigma_{0}$ is the initial cloud dimension.

Each WFM SDD tile has a geometric area of $77.4 \times 72.5 \mathrm{~mm}^{2}$, which corresponds to an active area of $65.1 \times 70.0 \mathrm{~mm}^{2}$. The detector thickness is $450 \mu \mathrm{m}$ and the anode pitch is $145 \mu \mathrm{m}$. Each SDD tile produces 2D images with a spatial resolution FWHM better than $70 \mu \mathrm{m}$ in the anode direction, and between $5 \mathrm{~mm}$ and $8 \mathrm{~mm}$ in the drift direction. The spectral resolution FWHM is $\sim 300 \mathrm{eV}$ at $6 \mathrm{keV}$ (End Of Life). For further details, see [5, 6, 7].

\section{Imaging simulations}

The imaging properties of two orthogonal Cameras (one Unit) have been studied in detail by means of a Monte Carlo simulator [8,9]. A simulated $10 \mathrm{ks}$ observation of the Galactic centre is shown in Figure 3. The map represents a central region $\left(\sim 60^{\circ} \times 30^{\circ}\right)$ of the FoV of one Unit obtained by combining the sky observed simultaneously by the two Cameras.

The performance of the LOFT Wide Field Monitor, as estimated by means of Monte Carlo simulations, are summarized in Table 2.

\section{LOFT and Gamma Ray Bursts}

The WFM will detect $\sim 150$ Gamma Ray Bursts per year. With a response down to $2 \mathrm{keV}$ and a good energy resolution the WFM will offer unique capabilities in this field of research. Moreover, LOFT will employ a VHF transmission capability to send a short message about the occurrence of such events. The LOFT burst alert system (LBAS) will distribute the location of a transient event 


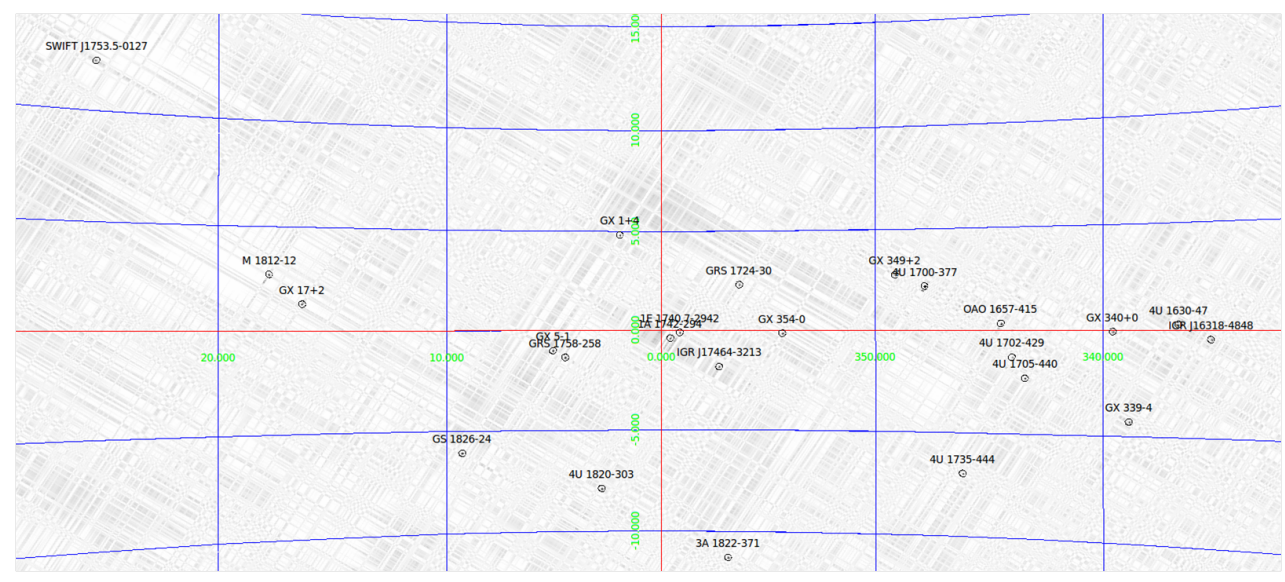

Figure 3: 10 ks observation (one WFM Unit) of the Galactic centre (central FoV region).

\begin{tabular}{l|ccc} 
Parameter & Camera & Unit & Overall WFM \\
\hline Energy range & \multicolumn{3}{|c}{$2-50 \mathrm{keV}$} \\
Active detector area & $182 \mathrm{~cm}^{2}$ & $364 \mathrm{~cm}^{2}$ & $1820 \mathrm{~cm}^{2}$ \\
Peak effective area & $45 \mathrm{~cm}^{2}$ & $90 \mathrm{~cm}^{2}$ & $90 \mathrm{~cm}^{2}$ \\
FoV Zero response & $90^{\circ} \times 90^{\circ}$ & $90^{\circ} \times 90^{\circ}$ & $210^{\circ} \times 90^{\circ}+90^{\circ} \times 90^{\circ}$ \\
FoV 20\% response & $60^{\circ} \times 60^{\circ}$ & $60^{\circ} \times 60^{\circ}$ & $180^{\circ} \times 60^{\circ}+60^{\circ} \times 60^{\circ}$ \\
Angular resolution (FWHM) & $5^{\prime} \times 5^{\circ}$ & $5^{\prime} \times 5^{\prime}$ & $5^{\prime} \times 5^{\prime}$ \\
Point Source Location Accuracy $(10 \sigma)$ & $<1^{\prime} \times 30^{\prime}$ & $<1^{\prime} \times 1^{\prime}$ & $<1^{\prime} \times 1^{\prime}$ \\
On-axis sens. $5 \sigma, 3 \mathrm{~s}, \mathrm{Gal}$ centre & $380 \mathrm{mCrab}$ & $270 \mathrm{mCrab}$ & $270 \mathrm{mCrab}$ \\
On-axis sens. $5 \sigma, 10 \mathrm{ks}$, Gal. centre & $6.6 \mathrm{mCrab}$ & $4.7 \mathrm{mCrab}$ & $4.7 \mathrm{mCrab}$ \\
\hline
\end{tabular}

Table 2: WFM performance.

with $\sim 1^{\prime}$ accuracy to end users within $30 \mathrm{~s}$ (goal $20 \mathrm{~s}$ ) of the on-board detection of the burst in at least $2 / 3$ of the cases. As an example of the WFM capabilities of detecting spectral features in the GRB prompt emission, in Figure 4 we report the simulated spectrum of the GRB 990705. The Figure shows how the transient absorption K-edge at $3.8 \mathrm{keV}$, detected by Beppo-SAX [10], would be probed by the LOFT/WFM.

\section{References}

[1] M. Feroci et al. The Large Observatory for X-ray Timing (LOFT). Experimental Astronomy, 34:415-444, October 2012.

[2] M. Feroci et al. LOFT: the Large Observatory For X-ray Timing. In Society of Photo-Optical Instrumentation Engineers (SPIE) Conference Series, volume 8443, September 2012.

[3] S. Brandt et al. The LOFT wide field monitor. In Society of Photo-Optical Instrumentation Engineers (SPIE) Conference Series, volume 8443, September 2012.

[4] G. K. Skinner. Sensitivity of coded mask telescopes. Applied Optics, 47:2739-2749, May 2008. 


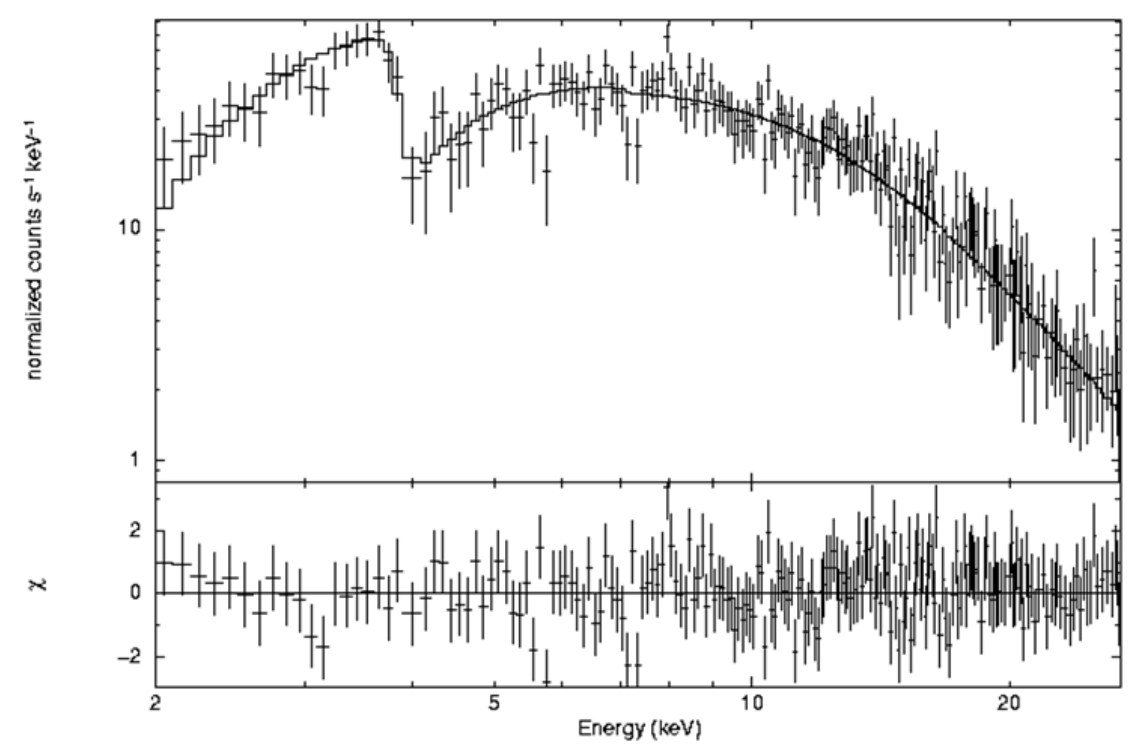

Figure 4: Simulated spectrum of the GRB 990705 prompt emission.

[5] R. Campana et al. Imaging performance of a large-area Silicon Drift Detector for X-ray astronomy. Nuclear Instruments and Methods in Physics Research A, 633:22-30, March 2011.

[6] G. Zampa et al. Room-temperature spectroscopic performance of a very-large area silicon drift detector. Nuclear Instruments and Methods in Physics Research A, 633:15-21, March 2011.

[7] Y. Evangelista et al. Simulations of the x-ray imaging capabilities of the silicon drift detectors (SDD) for the LOFT wide-field monitor. In Society of Photo-Optical Instrumentation Engineers (SPIE) Conference Series, volume 8443, September 2012.

[8] I. Donnarumma et al. The LOFT wide field monitor simulator. In Society of Photo-Optical Instrumentation Engineers (SPIE) Conference Series, volume 8443, September 2012.

[9] C. Schmid et al. LOFT Simulation Toolkit. In this proceedings, 2012.

[10] L. Amati et al. Discovery of a Transient Absorption Edge in the X-ray Spectrum of GRB 990705. Science, 290:953-955, November 2000. 